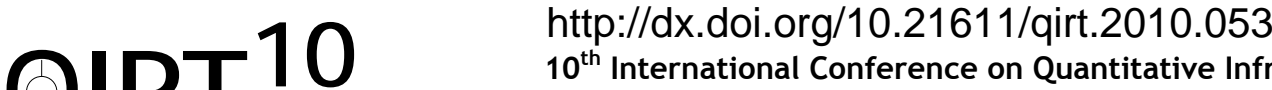 \\ $10^{\text {th }}$ International Conference on Quantitative InfraRed Thermography \\ July 27-30, 2010, Québec (Canada)
}

\section{Real-time monitoring of dental lesions using transmission-mode photothermal radiometry and modulated luminescence}

\author{
by A. Hellen ${ }^{* * *}$, A. Mandelis* ${ }^{*}$ Y. Finer** and B.T. Amaechi ${ }^{* * *}$
}

\author{
*Center for Advanced Diffusion Wave Technologies (CADIFT), MIE, University of Toronto; Toronto, ON, Canada \\ M5S 3G8; a.hellen@utoronto.ca; mandelis@mie.utoronto.ca \\ ${ }^{* \star}$ Faculty of Dentistry, University of Toronto; Toronto, ON, Canada M5G 1G6, yoav.finer@dentisty.utoronto.ca \\ ${ }^{* * * D e p a r t m e n t}$ of Community Dentistry, University of Texas Health Science Center at San Antonio, San Antonio \\ (Texas), USA7 8229-3900; Amaechi@uthscsa.edu
}

Dental caries is an infectious bacterial disease characterized by a dynamic equilibrium between cycles of acidinduced mineral loss (demineralization) originating in the dental hard tissue, and mineral recovery from inorganic ions at the tooth-saliva interface to restore form and function (remineralization). Dental enamel forms the outer layer of tooth tissue exposed to the oral environment and is composed of a calcium-deficient, carbonated hydroxyapatite ( $87 \%$ by volume). Weak acid challenges of dental enamel over time result in a demineralized lesion, coarsely manifested as a 3-layer geometrical profile with a relatively unaffected surface layer of high mineral volume overlying a region of subsurface mineral loss, where mineral volume may be as low as $50 \%$, followed by sound enamel [1]. The typical protocol employed in-vitro to study demineralization-remineralization phenomena often involves the use of multiple thin enamel sections each delineated with nail varnish to maintain a region of sound tissue, and interrupted repeatedly for analysis. As both demineralization and remineralization are time-dependent processes, significant sample interruption can affect both rate and extent of lesion formation [2]. Consequently, the ability to monitor changes as a function of time with minimal disturbance would be ideal. As an emerging non-destructive technique, frequency-domain photothermal radiometry (PTR) is an established sensitive methodology to characterize pathological dental tissues. PTR is based on the generation of diffuse-photon-density waves in turbid media by a harmonically modulated laser beam to induce an oscillatory temperature thermal-wave field, which can be detected with mid-IR detectors. Modulated luminescence (LUM) monitors the optical-to-radiative energy conversion, a complementary signal channel. The purpose of the present study was to evaluate the ability of PTR-LUM in transmission mode to monitor real-time changes in dental enamel during demineralization and remineralization processes.

The transmission-mode PTR-LUM setup consisted of a laser diode $(670 \mathrm{~nm}, 500 \mathrm{~mW})$, a mercury-cadmium-telluride (MCT) infrared detector and pre-amplifier for PTR and a photodiode for LUM. PTR and LUM signal channels were monitored through lock-in amplifiers. Enamel sections $(n=10)$ of about $1-1.5 \mathrm{~mm}$ thick, with undisturbed intact enamel surfaces, were polished on the cut face using successive grits of silicon carbide paper. Individual samples were fixed to one wall of a transparent container cut open to allow for transmitted IR signals to be collected, such that the tooth enamel surface was in contact with the treatment solution and normal to laser irradiation. Sections were subjected to demineralization in an acidified gel ( $\mathrm{pH} \mathrm{4.5)} \mathrm{for} 15$ days. PTR-LUM frequency scans immediately followed the addition of acidified gel to the individual containers fixed on the sample stage. Following demineralization, five samples were remineralized in a mineral solution (20 $\mathrm{mL}$ ) for 20 days. Intermittent PTR-LUM frequency scans $(1-1000 \mathrm{~Hz})$ were repeatedly performed throughout the treatment process without sample disruption. Transverse microradiography (TMR) analysis followed the conclusion of all treatments.

An exemplary artificial caries lesion, characterized by a thin, intact surface layer, superficial to a deep subsurface lesion body was created following demineralization and is shown in figure 1. Transmission PTR and LUM amplitude and phase signals revealed monotonic changes with increased demineralization time. A decrease in PTR amplitude occurred after $200 \mathrm{hrs}$. PTR amplitude and phase behaviour may be consistent with the formation or growth of a surface layer of
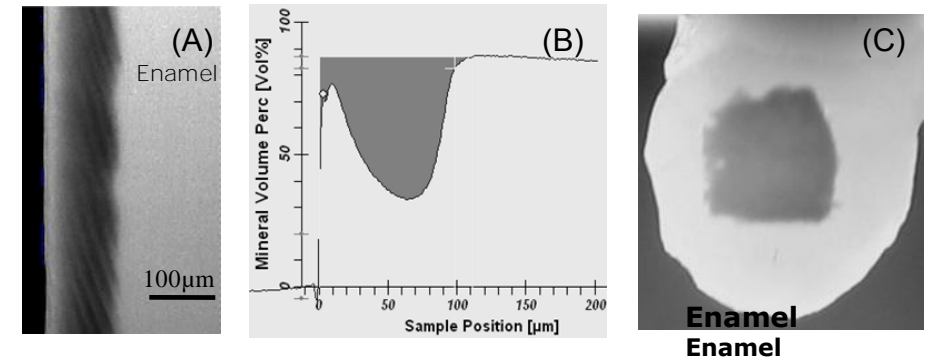

Fig. 1. Exemplary micrographic image (a), densitometric tracing (b). and visible liaht transmission image (c) of a demineralized enamel se 
poorer thermophysical properties with a slight increase in phase lag during the course of acid challenge. In addition, PTR phase lag increased at earlier demineralization times. Poorer properties would confine the thermal-wave centroid closer to the enamel surface. Changes in the optical and thermal transmission intensity reflect changes in absorption and scattering properties and thermal conductivities and diffusivities of the material. The decrease in PTR amplitude and increase in phase lag may further be related to enhanced lesion scattering properties during the demineralization process resulting in opticalthermal energy conversion in near-surface regions, farther away from the IR detector. The visible light transmission image of the thin demineralized enamel section is shown in figure 1c. The treated lesion zone is clearly discernible from the unaffected surrounding enamel. The increased phase lag seen at $1 \mathrm{~Hz}$, which offers the deepest penetration of the thermal-wave field, may reflect the depth of the lesion body, which extends deeper into enamel with greater acid exposure, at the expense of the underlying sound enamel tissue. LUM amplitudes and phase minima decreased monotonically with demineralization time. The loss of enamel mineral results in the loss of the tooth's autofluorescent properties. At the onset of remineralization, a step-decrease in phase lag at $1 \mathrm{~Hz}$ was accompanied by a concomitant increase in amplitude (figure 2 ). After $\approx 300$ hrs amplitude and phase signals reversed direction and reached stable values after $\approx 650 \mathrm{hrs}$ until the end of remineralization. This behaviour may reflect the mechanism of fluoride enhanced remineralization, promoting rapid

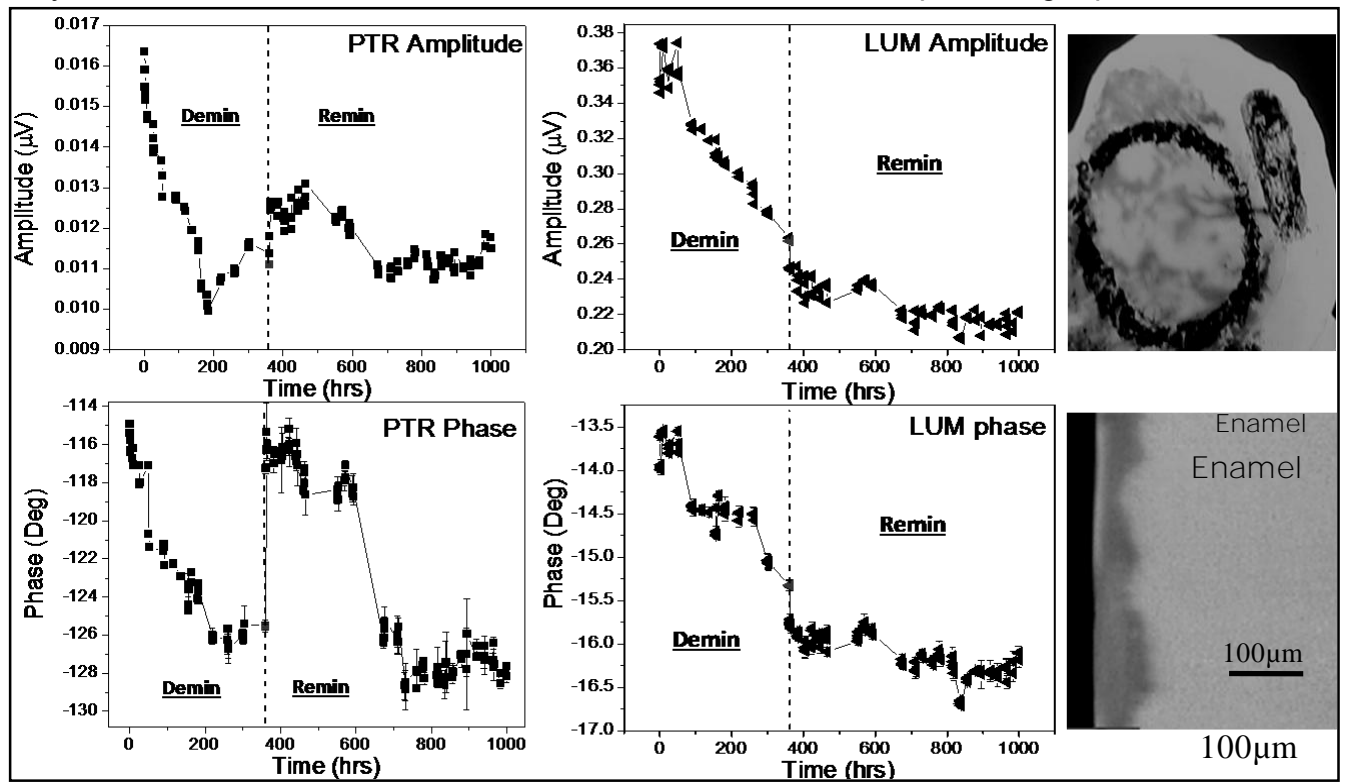

Fig. 2. Time-series PTR-LUM amplitude and phase signals at $1 \mathrm{~Hz}$ (PTR) and $89 \mathrm{~Hz}$ (LUM). Vertical dashed lines divide de-and remineralization treatments.

uptake and precipitation of mineral ions from solution within the entire lesion depth [3] with molecular out-diffusion and direct surface remineralization building up the surface remineralized region, similar to figure $1 \mathrm{~B}$, dominating the thermal-wave centroid at later remineralization times. This process may be enhanced for the first 10 days after remineralization, whereas surface mineral deposition may be dominant at later times. LUM amplitude and phases at $89 \mathrm{~Hz}$, the frequency where LUM phase minima appear, reveal similar trends as PTR however, exhibited less marked changes at the onset of remineralization. Visible light transmission images reveal the non-uniform appearance of remineralized enamel, with regions of mineral restoration intertwined with more opaque regions of demineralization.

The present study is the first account investigating real-time mineralization cycles in dental enamel using PTR-LUM in transmission and appears to be a valuable sensitive methodology to investigate mechanistic and kinetic aspects of de- and re-mineralization processes.

\section{REFERENCES}

[1] Kidd E.A.M. and Joyston-Bechal S., "Essentials of Dental Caries" $2^{\text {nd }}$ edition. New York: Oxford University Press, 1997.

[2] Gao X.J., Elliott J.C. and Anderson P., "Scanning microradiographic study of the kinetics of subsurface demineralization in tooth sections under constant-composition and small constant-volume conditions.," J Dent Res 72: 923-930, 1993.

[3] ten Cate J.M., "In vitro studies on the effects of fluoride on de- and remineralization.," J Dent Res 69 (Spec Iss):614619, 1990. 